\title{
Prevalência e fatores de risco de leptospirose bovina no Estado do Maranhão ${ }^{1}$
}

\author{
Felipe J. Silva ${ }^{2}$, Washington L.F. Conceição ${ }^{4}$, José J. Fagliari ${ }^{3}$, Raul J.S. Girio ${ }^{2}$, \\ Ricardo A. Dias ${ }^{5}$, Mauro R. Borba ${ }^{5}$ e Luis A. Mathias ${ }^{2}$
}

\begin{abstract}
Silva F.J., Conceição W.L.F., Fagliari J.J., Girio R.J.S., Dias R.A., Borba M.R. \& Mathias L.A. 2012. [Prevalence and risk factors of bovine leptospirosis in the State of Maranhão, Brazil.] Prevalência e fatores de risco de leptospirose bovina no Estado do Maranhão. Pesquisa Veterinária Brasileira 32(4):303-312. Departamento de Medicina Veterinária Preventiva e Reprodução Animal, Faculdade de Ciências Agrárias e Veterinárias, Universidade Estadual Paulista, Via de Acesso Professor Paulo Donato Castellane s/n, Zona Rural, Jaboticabal, SP 14884-900, Brazil. E-mail: fjsepi@gmail.com

Prevalence and risk factors of bovine leptospirosis in the State of Maranhão were investigated. Based on production parameters that vary across different production systems, management practices, the purpose of exploitation, the average size of herds and market systems, the state was divided in four sampling circuits. The study aimed to investigate the epidemiological features of bovine leptospirosis in the State of Maranhão, in order to determine the prevalence of the infection in cattle and herds, to determine the occurrence of serovars of Leptospira spp., to identify risk factors associated with leptospirosis in cattle and to differentiate the livestock circuits itself regarding the prevalence of leptospirosis. The survey was conducted in 136 herds in the circuit I, in which $841 \geq 24$ months old females were analyzed; 238 in the circuit II and 2,582 females were analyzed; 122 in the circuit III and 869 females were analyzed; 77 in the circuit IV and 540 females were analyzed; a total of 573 herds and 4,832 females were analyzed. The presence of antibodies against Leptospira spp. was verified by microscopic agglutination test (MAT). Of the 4,832 cows examined, 1,904 (35.94\%, CI $95 \%=33.01 \%-38.98 \%)$ were positive. Of the 573 herds, $380(64.81 \%$, CI $95 \%=61.10 \%$ $-68.35 \%$ ) were positive. Serovars Hardjo and Wolffi were the most frequent in the state. The circuit III showed the lowest prevalence of leptospirosis in all comparisons. The variables presence of horses $(p=0.000)$, presence of capybaras $(p=0.034)$ and herds with up to 32 adult females $(\mathrm{p}=0.002)$ were identified as risk factors for leptospirosis.

INDEX TERMS: Cattle, Maranhão, risk factors, leptospirosis, prevalence, microscopic agglutination.
\end{abstract}

\footnotetext{
${ }^{1}$ Recebido em 23 de julho de 2011.

Aceito para publicação em 28 de novembro de 2011.

${ }^{2}$ Departamento de Medicina Veterinária Preventiva e Reprodução Animal, Faculdade de Ciências Agrárias e Veterinárias (FCAV), Universidade Estadual Paulista (Unesp), Via de Acesso Professor Paulo Donato Castellane s/n, Zona Rural, Jaboticabal, SP 14884-900, Brasil. *Autor para correspondência: fjsepi@gmail.com

${ }^{3}$ Departamento de Clínica e Cirurgia Veterinária, FCAV-Unesp, Jaboticabal, SP.

${ }^{4}$ Departamento de Clínicas Veterinárias, Universidade Estadual do Maranhão, Cidade Universitária Paulo VI s/n, Tirirical, São Luís, MA 65055970, Brasil.

${ }^{5}$ Departamento de Medicina Veterinária Preventiva e Saúde Animal, Faculdade de Medicina Veterinária e Zootecnia, Universidade de São Paulo (USP), Av. Prof. Dr. Orlando Marques de Paiva 87, Cidade Universitária, São Paulo, SP 05508-270, Brasil.
}

RESUMO.- Foram investigados a prevalência e os fatores de risco da leptospirose bovina no Estado do Maranhão. 0 Estado foi dividido em quatro circuitos amostrais com base em parâmetros de produção distintos que variam conforme os diferentes sistemas de produção, as práticas de manejo, a finalidade de exploração, o tamanho médio dos rebanhos e os sistemas de comercialização. Objetivou-se estudar as características epidemiológicas da leptospirose bovina no Estado do Maranhão, de modo a determinar a prevalência em bovinos e em rebanhos, detectar as sorovariedades de Leptospira spp. presentes, identificar os fatores de risco eventualmente associados à leptospirose em bovinos e diferenciar os circuitos pecuários entre si no que se refere à prevalência de leptospirose. A pesqui- 
sa foi realizada em 136 propriedades rurais pertencentes ao circuito I, no qual 841 fêmeas bovinas com idade igual ou superior a 24 meses foram analisadas; 238 do circuito II, com 2.582 fêmeas analisadas; 122 do circuito III, com 869 fêmeas analisadas; e 77 do circuito IV, com 540 fêmeas analisadas; no total, 573 propriedades e 4.832 fêmeas foram estudadas. A presença de anticorpos contra Leptospira spp. foi verificada pela técnica de soroaglutinação microscópica (SAM). Das 4.832 fêmeas bovinas analisadas, 1.904 $(35,94 \%$; IC $95 \%=33,01 \%-38,98 \%)$ foram reagentes. Das 573 propriedades analisadas, $380(64,81 \%$; IC 95\% = $61,10 \%-68,35 \%$ ) foram consideradas positivas. As sorovariedades Hardjo e Wolffi foram as mais frequentes em todo o Estado. 0 circuito III foi o que apresentou menor prevalência de leptospirose em todas as comparações. As variáveis identificadas como fatores de risco de leptospirose foram: presença de equinos $(p=0,000)$, presença de capivaras $(p=0,034)$ e rebanhos bovinos com 32 ou mais fêmeas adultas $(p=0,002)$.

TERMOS DE INDEXAÇÃO: Bovinos, Estado do Maranhão, fatores de risco, leptospirose, prevalência, soroaglutinação microscópica.

\section{INTRODUÇÃO}

A leptospirose é uma importante zoonose em termos de saúde pública, com uma incidência elevada em períodos chuvosos, principalmente em regiões com baixos níveis socioeconômicos, nas quais a população humana tem maior contato com água de esgoto, inundações, ou seja, grandes chances de se infectar pelo contato com urina de animais infectados, disseminada facilmente pela água. Dados do Sistema Único de Saúde (SUS) apontam um gasto anual de $\mathrm{R} \$ 1,2$ milhão para o tratamento da leptospirose humana (Brasil, 2005).

Para a saúde animal, as consequências dessa infecção estão relacionadas à esfera econômica, tendo em vista o envolvimento de animais de produção como bovinos, ovinos, caprinos, suínos e equinos (Brasil 1995). Nessas espécies, as perdas econômicas decorrem principalmente de transtornos reprodutivos como infertilidade, abortamento, nascimento de crias fracas e diminuição temporária da produção leiteira (Cervantes et al. 2002).

Sua ocorrência está relacionada às precárias condições de infraestrutura sanitária, reflexo da baixa condição socioeconômica observada em nosso país (Brasil 2005). Além de ser uma doença de grande relevância para a saúde pública, é propensa a ocorrer em veterinários, trabalhadores de abatedouro e tratadores de animais, devido ao estreito contato com as fontes de infecção (Acha \& Szyfres 2001). As inundações favorecem a disseminação e a persistência do agente causal no ambiente, facilitando a ocorrência de surtos, uma vez que as águas das chuvas e dos rios misturam-se às águas de esgoto geralmente contaminadas com urina de roedores (Brasil 2005).

Estudos de prevalência da leptospirose em fêmeas bovinas e em rebanhos foram realizados em alguns estados brasileiros. Figueiredo et al. (2009) no Mato Grosso do Sul, Castro et al. (2008) em São Paulo e Oliveira et al. (2009) na Bahia relataram prevalência da leptospirose em fême- as bovinas de, respectivamente, $81,1 \%, 49,4 \%$ e $45,43 \%$. Homem et al. (2001) em Uruará, Aguiar et al. (2006) em Monte Negro, Figueiredo et al. (2009) no Mato Grosso do Sul, Oliveira et al. (2009) na Bahia e Castro et al. (2008) em São Paulo encontraram prevalência da leptospirose em rebanhos bovinos de, respectivamente, 97\%, 95,3\%, 90,4\%, $77,93 \%$ e $71,3 \%$.

Em diversas regiões do Brasil estudos demonstram prevalências em rebanhos bovinos que variam de $74 \%$ a $100 \%$ (Homem et al. 2001, Favero et al. 2001, Thompson et al, 2006, Lage et al. 2007) e, em animais, de 45,56\% a 62,3\% (Langoni et al. 2000, Favero et al. 2001). No Brasil, inquéritos sorológicos realizados em rebanhos bovinos evidenciam as sorovariedades Hardjo, Wolffi, Pomona, Grippotyphosa, Icterohaemorrhagiae e Canicola, sendo a sorovariedade Hardjo a mais prevalente (Favero et al. 2001, Lilenbaum \& Souza 2003, Araújo et al. 2005, Thompson et al. 2006, Lage et al. 2007). No Mato Grosso do Sul, a sorovariedade Hardjo também é a mais observada na sorologia (Madruga et al. 1980, Pellegrin et al. 1992, Pellegrin \& Sereno 1994, Pellegrin et al. 1999, Favero et al. 2001).

A profilaxia da leptospirose inclui a adoção de importantes procedimentos, como: controle de roedores, os quais são os maiores disseminadores do agente etiológico; eliminação do excesso de água estagnada no ambiente; isolamento e tratamento dos animais doentes; detecção e tratamento dos portadores sãos; e imunização sistemática dos animais (Guimarães et al. 1982/1983). Sobre os reservatórios, que são principalmente os roedores sinantrópicos, devem ser tomadas medidas de saneamento e antirratização, tais como destinação adequada do lixo, armazenamento correto dos alimentos de uso humano e animal, não armazenamento de entulhos, que servem como abrigo, e uso racional de rodenticidas (Brasil 1995).

A leptospirose humana é uma doença de notificação compulsória no Brasil (Brasil 2005). A leptospirose animal possui grande importância do ponto de vista socioeconômico e sanitário, tendo considerável repercussão no comércio internacional de animais e seus produtos (Blaha 1989).

Mesmo existindo um grande número de trabalhos sobre a frequência de bovinos reagentes à leptospirose bovina em diversos estados brasileiros, são poucos os que utilizam amostras aleatórias. Além disso, ainda não há estudos sobre a prevalência e os fatores de risco de leptospirose bovina no Estado do Maranhão. Por isso, o objetivo do trabalho foi estudar as características epidemiológicas da leptospirose bovina nesse Estado.

\section{População estudada}

\section{MATERIAL E MÉTODOS}

Foi utilizado o banco de soro oriundo do estudo da situação epidemiológica da brucelose bovina no Estado do Maranhão, realizado como parte do Programa Nacional de Controle e Erradicação da Brucelose e Tuberculose e planejado pelo Ministério da Agricultura, Pecuária e Abastecimento (MAPA), em colaboração com a Agência Estadual de Defesa Agropecuária do Estado da Maranhão (AGED-MA). Nesse estudo foram analisados 4.832 soros de fêmeas bovinas com idade igual ou superior a 24 meses, provenientes de 573 propriedades, sorteadas dentro de quatro estratos 
amostrais do Estado. Em propriedades com até 99 fêmeas com idade igual ou superior a 24 meses foram amostradas 10 delas ou todas as fêmeas dessa faixa etária, se elas fossem menos do que dez. Naquelas com 100 ou mais foram amostradas 15 delas.

\section{Marco amostral}

A ocorrência da leptospirose foi estimada por um processo amostral aleatório que detectou a prevalência de rebanhos e de animais com a infecção, e os resultados foram extrapolados para cada circuito estudado. Em cada circuito, o tamanho da amostra foi determinado pelo grau de confiança do resultado, pelo nível de precisão desejado e pelo valor de prevalência esperado (Noordhuizen et al. 1997) e utilizando a fórmula para amostra simples aleatória, de acordo com Thrusfield (1995) e Noordhuizen et al. (1997):

$$
\mathrm{n}=\frac{\mathrm{Z}^{2}}{\alpha} \cdot \frac{\sqrt{\mathrm{p}}(1-\mathrm{p})}{\mathrm{d}^{2}}
$$

Em que:

$\mathrm{n}=$ número de propriedades amostradas por circuito produtor;

$\mathrm{Z}_{\alpha}=$ valor da distribuição normal para o grau de confiança de $95 \%$;

$\mathrm{p}=$ prevalência esperada, fixada em $20 \%$;

$\mathrm{d}=$ erro absoluto, fixado em $6 \%$.

Esses cálculos serviram de base para obtenção das unidades secundárias de amostragem.

\section{Preparo dos antígenos de Leptospira spp.}

As sorovariedades de Leptospira spp. utilizadas como antígenos, Australis, Bratislava, Autumnalis, Butembo, Castellonis, Bataviae, Canicola, Whitcombi, Cynopteri, Grippotyphosa, Hebdomadis, Copenhageni, Icterohaemorrhagiae, Javanica, Panama, Pomona, Pyrogenes, Hardjo, Wolffi, Shermani, Tarassovi, Andamana, Patoc, Sentot, foram provenientes de matrizes, replicadas semanalmente em meio de cultura líquido de EMJH (Ellighausen, McCullough, Johnson e Harris - Difco ${ }^{\mathrm{R}}$ ), tendo como inóculo 10\% do volume do meio a semear, e mantidas em estufa bacteriológica BOD a $28^{\circ} \mathrm{C}$. Todos os antígenos foram utilizados ao redor do sexto dia de incubação. Foram utilizados apenas antígenos puros. A concentração considerada ideal foi padronizada de forma a corresponder à metade da turvação do tubo número 1 da escala de MacFarland (cerca de 100 a 200 leptospiras por campo microscópico), segundo a técnica de Sulzer \& Jones (1980). As culturas estavam isentas de contaminação e de autoaglutinação.

\section{Técnica de soroaglutinação microscópica (SAM).}

As amostras de soro, devidamente armazenadas e congeladas, foram encaminhadas ao Laboratório de Diagnóstico de Brucelose e Leptospirose do Departamento de Medicina Veterinária Preventiva e Reprodução Animal, da Faculdade de Ciências Agrárias e Veterinárias de Jaboticabal (FCAV-Unesp).

A presença de anticorpos contra Leptospira spp. nos soros foi verificada pela SAM, a partir de uma diluição 1/50, sendo uma parte de soro sanguíneo para 49 partes de solução salina $0,85 \%$. Dessa diluição foram colocadas alíquotas de $25 \mu \mathrm{L}$ em placas de polietileno, com fundo plano, e adicionada igual quantidade do antígeno, o que resultou em uma diluição $1 / 100$. As placas foram então incubadas em estufa bacteriológica BOD à temperatura de $28^{\circ} \mathrm{C}$ por uma hora. A leitura foi realizada em microscópio de campo escuro, diretamente na placa, utilizando a objetiva de $10 \mathrm{x}$ e ocular de 10x.

0 critério adotado para considerar um soro como reagente foi a aglutinação de pelo menos $50 \%$ das leptospiras no campo microscópico no aumento de 100x. Os soros reagentes na triagem inicial foram reexaminados em sete diluições seriadas de razão dois e selecionadas apenas as amostras com titulação mínima de 100 (ponto-de-corte), ou seja, todas as reagentes (Santa Rosa et al. 1975, Santa Rosa et al. 1980).

\section{Questionário e análise dos fatores de risco}

Durante as colheitas de sangue, foram realizadas entrevistas com aplicação de questionário investigativo, desenvolvido pela AGED-MA e pelo MAPA, contendo perguntas referentes a sistema de produção, presença de ovinos/caprinos, presença de equinos, presença de suínos, presença de cães, presença de animais selvagens, presença de cervídeos, presença de roedores, presença de outros animais selvagens, venda de reprodutores, compra de reprodutores, aluguel de pasto, pasto em comum com outras propriedades, áreas alagadiças nas pastagens, piquete de parição e assistência veterinária. As entrevistas e os questionários foram aplicados por pessoa treinada anteriormente para realizar essa tarefa.

As variáveis de risco associadas à soropositividade do rebanho para pelo menos uma sorovariedade foram analisadas por meio da estimativa pontual e intervalar da odds ratio (OR), com intervalo de confiança de $95 \%$. 0 teste de hipóteses foi realizado com o teste de qui-quadrado $\left(\mathrm{x}^{2}\right)$ ou o teste exato de Fisher, quando indicado (Sampaio 2007).

\section{Prevalência de focos de leptospirose bovina no Estado do Ma- ranhão}

Para o cálculo da prevalência de focos de leptospirose no Estado do Maranhão, foi considerada a amostra aleatória estratificada como desenho amostral, e cada um dos quatro circuitos foi considerado um estrato.

Os parâmetros necessários para o cálculo foram: o diagnóstico (positivo ou negativo) da propriedade, a área da propriedade e o peso estatístico (Quadro 2). Este último foi calculado da seguinte forma:

Peso estatístico $=\frac{\mathrm{n}^{\mathrm{o}} \text { total de propriedades no estrato }}{\mathrm{n}^{\mathrm{o}} \text { de propriedades amostradas no estrato }}$

Portanto, para o cálculo da prevalência de focos de leptospirose, tem-se:

Prevalência $=\frac{\mathrm{n} \text { o de propriedades foco } \mathrm{x} \text { peso estatístico }}{\mathrm{n} \text { o de propriedades analisadas }}$

Foi considerada a amostra aleatória simples como desenho amostral para o cálculo da prevalência de focos de leptospirose bovina em cada um dos quatro circuitos do Estado do Maranhão.

Os parâmetros necessários para o cálculo foram o número de focos e o número de propriedades amostradas na área.

Foi considerada foco a propriedade que continha pelo menos quatro animais sororreagentes a leptospirose, ou seja, o achado de quatro ou mais animais reagentes no rebanho classificava a propriedade como positiva, de um a três animais como inconclusiva, e nenhum animal reagente, como negativa (Figueiredo et al. 2009).

\section{Prevalência de leptospirose em animais no Estado do Mara- nhão}

Para o cálculo da prevalência de leptospirose em animais, foi considerada a amostra estratificada e por "cluster" como desenho amostral. Em cada um dos quatro circuitos, o desenho amostral considerado, para o cálculo de prevalência, foi a amostragem por "cluster".

Cada um dos quatro circuitos do Estado do Maranhão foi considerado como sendo um estrato, e cada propriedade como sendo um "cluster".

Os parâmetros utilizados foram: o diagnóstico (positivo ou 
negativo) do animal, a área a que o animal pertence e o peso estatístico. Este último foi calculado da seguinte forma:

Peso estatístico $=$ fração $\mathrm{A} \times$ fração $\mathrm{B}$

Fração $\mathrm{A}=\quad \mathrm{n}$ ㅇ total de fêmeas $\geq 24$ meses no estrato $\mathrm{n}$ - total de fêmeas $\geq 24$ meses nas propriedades amostradas no estrato

Fração $\mathrm{B}=\quad \mathrm{n}$ o total de fêmeas $\geq 24$ meses na propriedade no total de fêmeas $\geq 24$ meses amostradas na propriedade

A fração A indica o número de animais que cada animal selecionado em uma propriedade representa na população.

A fração B indica o número de animais que cada animal selecionado representa na propriedade.

O Quadro 1 relaciona os circuitos produtores do Estado do Maranhão e seus respectivos pesos estatísticos.

Portanto, para o cálculo da prevalência de leptospirose em animais, tem-se:

Prevalência $=\underline{n-0}$ de animais reagentes $x$ peso estatístico (fração $A \times$ fração $B$ ) $\mathrm{n}^{\mathrm{o}}$ de animais analisados

Quadro 1. Circuitos produtores do Estado do Maranhão e seus respectivos pesos estatísticos. Maranhão (2005-2009)

\begin{tabular}{cc}
\hline Circuito & Peso estatístico \\
\hline I & 139,3897 \\
II & 197,3739 \\
III & 204,4016 \\
IV & 133,9091
\end{tabular}

Diferença entre os circuitos pecuários no que se refere à prevalência da leptospirose em bovinos e em rebanhos do Estado do Maranhão

As diferenças na prevalência de anticorpos contra Leptospira spp. em fêmeas bovinas com idade igual ou maior que 24 meses e em rebanhos entre os circuitos produtores do Estado do Maranhão foram calculadas pelo método qui-quadrado $\left(\mathrm{x}^{2}\right)$ (Sampaio 2007). 0 valor exato de "p" foi obtido com o programa Excel 2010.

\section{Estudo dos fatores de risco de leptospirose bovina}

As variáveis de caracterização epidemiológica permitiram identificar os fatores de risco de ocorrência de leptospirose bovina nas propriedades. Foram estudados como possíveis fatores de risco: tipo de exploração ou sistema de produção (carne, leite, misto), tipo de criação (intensivo, semiextensivo, extensivo), número de bovinos na propriedade, prática de inseminação artificial, presença de outras espécies domésticas, presença de animais silvestres, compra de animais, abate de reprodutores, aluguel de pastos, pastos comuns com outras propriedades, piquete de parição, pastos alagados e assistência veterinária.

Inicialmente foi realizada uma análise univariada, utilizando-se os testes qui-quadrado $\left(\mathrm{x}^{2}\right)$ e exato de Fisher, a fim de verificar a associação de cada um dos possíveis fatores de risco com a variável dependente (rebanho livre ou infectado por leptospirose). Posteriormente foi realizada uma análise multivariada, na qual foi construído um modelo de regressão ( $\mathrm{y}=\mathrm{B} 0+\mathrm{B} 1 \mathrm{x}+\mathrm{B} 2 \mathrm{x}+\mathrm{B} 2 \mathrm{x} \ldots$.$) .$ Quando se insere uma variável, ela não está sozinha no modelo (interage com a variável B0 = intersecção). A partir daí, todas as variáveis inseridas interagem com as variáveis presentes no modelo (Sampaio 2007).

\section{RESULTADOS}

Das 4.832 fêmeas bovinas analisadas no Estado do Maranhão, 1.904 (35,94\%; IC 95\% = 33,01\% - 38,98\%) foram reagentes na SAM a pelo menos uma sorovariedade de Leptospira spp., com títulos variando de 100 a 6.400 .

No circuito I, das 841 fêmeas bovinas analisadas, 499 (58,30\%; IC $95 \%=53,16 \%-63,28 \%)$ foram positivas. No circuito II, das 2.582 fêmeas analisadas, 973 (33,41\%; IC $95 \%=29,49 \%-37,58 \%$ ) foram reagentes. No circuito III, das 869 fêmeas analisadas, 153 (15,53\%; IC 95\% = 11,05\% - 21,39\%) foram positivas. No circuito IV, das 540 fêmeas analisadas, 279 (63,14\%; IC 95\% = 56,84\% - 69,03\%) foram reagentes. Esses dados estão representados no Quadro 2.

Observa-se, pela análise do Quadro 2, maior soroprevalência em fêmeas bovinas no circuito IV, seguido pelo circuito I, circuito II e circuito III.

Quadro 2. Soroprevalência (animais reagentes na SAM a pelo menos uma sorovariedade) de Leptospira spp. em fêmeas bovinas em cada circuito produtor do Estado do Maranhão (2005-2009)

\begin{tabular}{|c|c|c|c|c|}
\hline \multirow{2}{*}{$\begin{array}{l}\text { Circuito } \\
\text { produtor }\end{array}$} & \multicolumn{2}{|c|}{ Animais } & \multirow{2}{*}{$\begin{array}{c}\text { Prevalência } \\
(\%)\end{array}$} & \multirow[t]{2}{*}{ IC 95\% (\%) } \\
\hline & Analisados & Reagentes & & \\
\hline I & 841 & 499 & 58,30 & {$[53,16-63,28]$} \\
\hline II & 2.582 & 973 & 33,41 & {$[29,49-37,58]$} \\
\hline III & 869 & 153 & 15,53 & {$[11,05-21,39]$} \\
\hline IV & 540 & 279 & 63,14 & {$[56,84-69,03]$} \\
\hline MA & 4.832 & 1.904 & 35,94 & {$[33,01-38,98]$} \\
\hline
\end{tabular}

As diferenças entre as prevalências de leptospirose em fêmeas bovinas do circuito I e do circuito II, do I e do III, do II e do III, do II e do IV, do circuito III e do circuito IV foram significativas $\left(\mathrm{p}=5,09 \times 10^{-28} ; \mathrm{p}=3,44 \times 10^{-70} ; \mathrm{p}=1,47 \times\right.$ $10^{-27} ; \mathrm{p}=2,22 \times 10^{-9}$ e $\mathrm{p}=4,54 \times 10^{-41}$, respectivamente). A diferença entre as prevalências do circuito I e do circuito IV também foi significativa ( $\mathrm{p}=0,006)$.

Das 573 propriedades analisadas, 380 (64,81\%; IC 95\% $=61,10 \%-68,35 \%$ ) foram consideradas positivas, ou seja, foram propriedades que tiveram quatro ou mais fêmeas bovinas reagentes a pelo menos uma sorovariedade de Leptospira spp., com títulos variando de 100 a 6.400 .

No circuito I, das 136 propriedades analisadas, 101 $(74,26 \%$; IC $95 \%=66,22 \%-80,95 \%)$ foram positivas. No circuito II, das 238 propriedades analisadas, 183 (76,89\%; IC $95 \%=71,08 \%-81,83 \%$ ) foram positivas. No circuito III, das 122 propriedades analisadas, 37 (30,33\%; IC 95\% = 22,79\% - 39,10\%) foram positivas. No circuito IV, das 77 propriedades analisadas, 59 (76,62\%; IC 95\% = 65,81\% - 84,81\%) foram positivas. Esses dados estão representados no Quadro 3.

Pela análise do Quadro 3, nota-se que houve maior soroprevalência em rebanhos no circuito II, seguido pelo circuito IV, pelo circuito I e pelo circuito III. Ainda na mesma análise, nota-se que o circuito II passou a ser o de maior soroprevalência em rebanhos, enquanto o circuito IV foi o de maior soroprevalência em fêmeas bovinas. 0 circuito III foi o de menor soroprevalência tanto em rebanhos quanto em fêmeas bovinas.

As diferenças entre as prevalências de leptospirose em rebanhos do circuito I e do circuito II, do I e do IV, do II e do IV não foram significativas ( $p=0,66$; $p=0,84$; e $p=0,89$, respectivamente). Já as diferenças entre as prevalências do circuito I e do circuito III, do II e do III, do III e do IV foram 
Quadro 3. Soroprevalência (pelo menos quatro animais reagentes na SAM) de Leptospira spp. em rebanhos em cada circuito produtor do Estado do Maranhão (2005 - 2009)

\begin{tabular}{ccccc}
\hline Circuito & \multicolumn{2}{c}{ Propriedades } & Prevalência (\%) & IC 95\% (\%) \\
\cline { 2 - 3 } produtor & Analisadas & Reagentes & & \\
\hline I & 136 & 101 & 74,26 & {$[66,22-80,95]$} \\
II & 238 & 183 & 76,89 & {$[71,08-81,83]$} \\
III & 122 & 37 & 30,33 & {$[22,79-39,10]$} \\
IV & 77 & 59 & 76,62 & {$[65,81-84,81]$} \\
MA & 573 & 380 & 64,81 & {$[61,10-68,35]$}
\end{tabular}

significativas $\left(p=3,94 \times 10^{-12} ; p=7,16 \times 10^{-16}\right.$ e $p=4,99 \times$ $10^{-10}$ ).

A sorovariedade Hardjo foi a mais encontrada no Estado do Maranhão (24,32\%) entre os animais reagentes na SAM, seguida pelas sorovariedades Wolffi $(22,00 \%)$, Patoc $(12,42 \%)$, Shermani $(8,85 \%)$, Grippotyphosa $(8,21 \%)$, Hebdomadis (7,35\%), Tarassovi (2,96\%), Autumnalis $(2,87 \%)$, Pyrogenes $(2,58 \%)$, Pomona $(2,26 \%)$, Copenhageni $(1,59 \%)$, Australis $(1,24 \%)$, Canicola $(1,15 \%)$, Castellonis $(1,05 \%)$, Icterohaemorrhagiae $(0,48 \%)$, Panama $(0,38 \%)$, Sentot $(0,22 \%)$ e Andamana $(0,06 \%)$.

A sorovariedade Icterohaemorrhagiae teve pouca ocorrência em fêmeas bovinas no Estado do Maranhão. Já as sorovariedades Hardjo e Wolffi foram as que tiveram maior ocorrência entre os animais reagentes $(24,32 \%$ e $22,00 \%$, respectivamente).

Das 4.832 fêmeas bovinas analisadas, 989 (20,52\%; IC $95 \%=17,94 \%-23,37 \%$ ) foram reagentes na SAM às sorovariedades Hardjo e/ou Wolffi, com títulos variando de 100 a 6.400 .

No circuito I, das 841 fêmeas bovinas analisadas, 347 $(41,63 \%$; IC $95 \%=36,66 \%-46,78 \%)$ foram reagentes às sorovariedades Hardjo e/ou Wolffi. No circuito II, das 2.582 fêmeas analisadas, 523 (22,20\%; IC 95\% = 18,58\% - 26,30\%) foram reagentes. No circuito III, das 869 fêmeas analisadas, 26 (2,63\%; IC 95\% = 0,96\% - 6,99\%) foram positivas. No circuito IV, das 540 fêmeas analisadas, 93 $(20,97 \%$; IC $95 \%=16,14 \%-26,79 \%)$ foram reagentes. Esses dados estão representados no Quadro 4.

Observa-se, pela análise do Quadro 4, que houve maior soroprevalência das sorovariedades de Leptospira spp. Hardjo e/ou Wolffi em fêmeas bovinas no circuito I, seguido pelo circuito II, pelo circuito IV e pelo circuito III.

As diferenças entre as prevalências das sorovariedades Hardjo e/ou Wolffi em fêmeas bovinas do circuito I e do circuito II, do I e do III, do I e do IV, do II e do III, do III e do IV foram significativas $\left(\mathrm{p}=9,89 \times 10^{-34} ; \mathrm{p}=2,6 \times 10^{-81} ; \mathrm{p}=1,44\right.$ $\mathrm{x} 10^{-20} ; \mathrm{p}=4,43 \times 10^{-33}$ e $\mathrm{p}=2,46 \times 10^{-20}$, respectivamente).

Quadro 4. Animais positivos na SAM às sorovariedades Hardjo e/ou Wolffi do total de animais testados e prevalência dessas sorovariedades nos animais em cada circuito produtor do Estado do Maranhão (2005-2009)

\begin{tabular}{ccccc}
\hline \multirow{2}{*}{$\begin{array}{c}\text { Circuito } \\
\text { produtor }\end{array}$} & \multicolumn{2}{c}{ Animais } & Prevalência (\%) & IC 95\% (\%) \\
\cline { 2 - 3 } & Analisados & Reagentes & & \\
\hline I & 841 & 347 & 41,63 & {$[36,66-46,78]$} \\
II & 2.582 & 523 & 22,20 & {$[18,58-26,30]$} \\
III & 869 & 26 & 2,63 & {$[0,96-6,99]$} \\
IV & 540 & 93 & 20,97 & {$[16,14-26,79]$} \\
MA & 4.832 & 989 & 20,52 & {$[17,94-23,37]$}
\end{tabular}

Já a diferença entre as prevalências do circuito II e do circuito IV não foi significativa $(p=0,12)$.

Das 573 propriedades analisadas, 227 (38,18\%; IC 95\% $=34,66 \%-41,84 \%$ ) foram reagentes às sorovariedades Hardjo e/ou Wolffi, com títulos variando de 100 a 6.400.

No circuito I, das 136 propriedades analisadas, 78 $(57,35 \%$; IC $95 \%=34,57 \%-51,14 \%)$ foram positivas. No circuito II, das 238 propriedades analisadas, 120 (50,42\%; IC $95 \%=44,07 \%-56,76 \%$ ) foram positivas. No circuito III, das 122 propriedades analisadas, 4 (3,28\%; IC $95 \%=1,23 \%$ - 8,46\%) foram positivas. No circuito IV, das 77 propriedades analisadas, 25 (32,47\%; IC 95\% = 22,91\% - 43,75\%) foram positivas. Esses dados estão representados no Quadro 5.

Nota-se, pela análise do Quadro 5, que houve maior soroprevalência das sorovariedades de Leptospira spp. Hardjo e/ou Wolffi em rebanhos no circuito I, seguido pelo circuito II, pelo circuito IV e pelo circuito III. Observa-se,

Quadro 5. Propriedades positivas na SAM às sorovariedades Hardjo e/ou Wolffi do total de propriedades testadas e prevalência dessas sorovariedades nos rebanhos em cada circuito produtor do Estado do Maranhão (2005-2009)

\begin{tabular}{ccccc}
\hline \multirow{2}{*}{$\begin{array}{c}\text { Circuito } \\
\text { produtor }\end{array}$} & \multicolumn{2}{c}{ Propriedades } & Prevalência (\%) & IC 95\% (\%) \\
\cline { 2 - 3 } & Analisadas & Reagentes & & \\
\hline I & 136 & 78 & 57,35 & {$[34,57-51,14]$} \\
II & 238 & 120 & 50,42 & {$[44,07-56,76]$} \\
III & 122 & 4 & 3,28 & {$[1,23-8,46]$} \\
IV & 77 & 25 & 32,47 & {$[22,91-43,75]$} \\
MA & 573 & 227 & 38,18 & {$[34,66-41,84]$}
\end{tabular}

ainda na mesma análise, a baixa soroprevalência das sorovariedades de Leptospira spp. Hardjo e/ou Wolffi em fêmeas bovinas e em rebanhos no circuito III.

A diferença entre as prevalências das sorovariedades Hardjo e/ou Wolffi em rebanhos do circuito I e do circuito II não foi significativa $(\mathrm{p}=0,23)$. As diferenças entre as prevalências das sorovariedades Hardjo e/ou Wolffi do circuito I e do circuito III, do I e do IV, do II e do III, do circuito III e do circuito IV foram significativas $\left(p=4,33 \times 10^{-20} ; p=8,1\right.$ $\mathrm{x} 10^{-4} ; \mathrm{p}=1,46 \times 10^{-18}$ e $\left.\mathrm{p}=4,32 \times 10^{-8}\right)$. A diferença entre as prevalências do circuito II e do circuito IV também foi significativa $(\mathrm{p}=0,009)$.

0 Quadro 6 demonstra a análise univariada feita para os possíveis fatores de risco de leptospirose bovina em rebanhos com atividade reprodutiva no Estado do Maranhão. Somente por essa análise não se pode concluir o que, de fato, é fator de risco, pois todas essas variáveis não se apresentam de maneira separada no ambiente, estão juntas e interagem no sistema. Portanto, nesse caso, para a análise conclusiva dos fatores de risco de leptospirose bovina no Estado do Maranhão, foi feita uma análise multivariada, representada no Quadro 7, e por sua análise, observa-se que as variáveis presença de equinos, presença de capivaras e rebanhos bovinos com fêmeas com idade igual ou superior a 24 meses acima de 32 cabeças foram identificadas como fatores de risco de leptospirose $(\mathrm{p}<0,05)$.

\section{DISCUSSÃO}

A prevalência de 35,94\% (33,01\% - 38,98\%) de fêmeas bovinas reagentes na SAM a pelo menos uma sorovariedade 
Quadro 6. Análise univariada dos possíveis fatores de risco de leptospirose bovina em rebanhos com atividade reprodutiva no Estado do Maranhão (2005-2009)

\begin{tabular}{|c|c|c|c|}
\hline Variável & Casos/Expostos & Casos/Não expostos & Valor de $\mathrm{p}$ \\
\hline Exploração de corte ${ }^{a}$ & $162 / 256$ & $215 / 313$ & 0,175 \\
\hline Exploração de leite ${ }^{\mathrm{b}}$ & $46 / 70$ & $331 / 499$ & 0,918 \\
\hline Exploração mistac $^{c}$ & $169 / 243$ & $208 / 326$ & 0,152 \\
\hline Criação extensiva ${ }^{\mathrm{d}}$ & $92 / 152$ & $286 / 419$ & 0,084 \\
\hline Inseminação artificiale & $12 / 17$ & $363 / 550$ & 0,694 \\
\hline Presença de ovinos e caprinos & $95 / 125$ & $285 / 448$ & 0,010 \\
\hline Presença de eqüinos & $312 / 429$ & $68 / 144$ & 0,000 \\
\hline Presença de suínos & $153 / 220$ & $227 / 353$ & 0,197 \\
\hline Presença de cão & $280 / 406$ & $100 / 167$ & 0,037 \\
\hline Presença de animais silvestres & $33 / 50$ & $347 / 523$ & 0,960 \\
\hline Presença de cervídeos & $54 / 76$ & $326 / 497$ & 0,348 \\
\hline Presença de capivaras & $24 / 27$ & $356 / 546$ & 0,011 \\
\hline Presença de outros silvestres & $27 / 30$ & $353 / 543$ & 0,005 \\
\hline Compra de reprodutores & $230 / 324$ & $149 / 248$ & 0,006 \\
\hline Abate de reprodutores ${ }^{f}$ & $71 / 114$ & $306 / 456$ & 0,330 \\
\hline Aluguel de pastos & $93 / 133$ & $286 / 439$ & 0,307 \\
\hline Presença de pasto comum & $98 / 142$ & $280 / 427$ & 0,452 \\
\hline Presença de áreas alagadiças & $184 / 283$ & $192 / 285$ & 0,554 \\
\hline Ausência de piquete de parição & $227 / 376$ & $150 / 194$ & 0,000 \\
\hline Ausência de assistência veterinária & $311 / 469$ & $59 / 88$ & 0,894 \\
\hline Rebanhos bovinos $>83$ animais $^{g}$ & $151 / 187$ & $229 / 386$ & 0,000 \\
\hline $\begin{array}{l}\text { Rebanhos bovinos com fêmeas > } 24 \text { meses } \\
\text { (acima de } 32 \text { animais) }^{g}\end{array}$ & $153 / 189$ & $227 / 384$ & 0,000 \\
\hline $\begin{array}{l}\text { a leite + misto x corte (risco). } \\
{ }^{b} \text { corte + misto x leite (risco). } \\
{ }^{c} \text { corte + leite x misto (risco). } \\
{ }^{\text {d }} \text { confinado + semiconfinado x extensivo (ris } \\
{ }^{\text {e }} \text { não usa IA x usa IA + usa IA e touro (risco). } \\
{ }^{\text {f }} \text { não abate + abate com inspeção + abate ser } \\
\text { g } 2^{o} \text { tercil. }\end{array}$ & co). & enda (risco). & \\
\hline
\end{tabular}

Quadro 7. Modelo final de regressão logística multivariada para os possíveis fatores de risco de leptospirose bovina em rebanhos com atividade reprodutiva no Estado do Maranhão (2005-2009)

\begin{tabular}{lccc}
\hline \multicolumn{1}{c}{ Variáveis } & Valor de p & Odds Ratio & IC 95\% (\%) \\
\hline Presença de eqüinos & 0,000 & 2,231 & {$[1,477-3,370]$} \\
Presença de capivaras & 0,034 & 3,817 & {$[1,110-13,132]$} \\
Rebanhos bovinos com fême- & 0,002 & 2,069 & {$[1,312-3,262]$}
\end{tabular}

as $\geq 24$ meses (acima de 32

animais)

$r^{2}=12,8 \%$.

de Leptospira spp. foi bastante alta, porém inferior às encontradas em outros estudos. Figueiredo et al. (2009) encontraram, no Estado do Mato Grosso do Sul, 81,1\% (76,3\% - 85,9\%) de fêmeas bovinas reagentes. Ainda na região Centro-Oeste, estudos realizados por Juliano et al. (2000), em bovinos leiteiros na microrregião de Goiânia, e por Madruga et al. (1980), em bovinos de corte no Estado do Mato Grosso, demonstraram prevalência em animais de 81,9\% e $74,5 \%$, respectivamente, o que sugere que a infecção por Leptospira spp. se mantém em alta prevalência há décadas nessa região, independentemente do tipo de exploração.

Alta prevalência também foi observada no estudo de Favero et al. (2001), em 21 estados brasileiros, no qual foi verificada uma proporção de $62,3 \%$ de animais reagentes. Especificamente no Estado do Maranhão, Favero et al. (2001) encontraram 58,2\% de bovinos reagentes a pelo menos uma sorovariedade, sendo desses $42,3 \%$ a Hardjo e $33 \%$ a Wolffi; $91,6 \%$ das propriedades e $100 \%$ dos muni- cípios analisados foram positivos nesse estado. Langoni et al. (2000), em bovinos diferentes regiões do Estado de São Paulo, registraram $45,56 \%$ de prevalência. Nesse mesmo Estado, Castro et al. (2008) encontraram soroprevalência de 49,4\% (44,4\% - 54,4\%) nas fêmeas bovinas em idade reprodutiva. Ainda no Estado de São Paulo, resultado inferior ao da presente investigação foi verificado por Martins (2005), num estudo da situação epidemiológica da leptospirose bovina, canina e humana nas propriedades rurais do Município de Pirassununga, onde foi encontrada prevalência de $30,3 \%(28,4-32,2)$ em bovinos e $88,4 \%(79,7-94,3)$ em rebanhos.

No Estado da Bahia, Oliveira et al. (2009) relataram soroprevalência de 45,43\% (42\% - 48,88\%) de animais soropositivos, e no Estado de Pernambuco, Oliveira et al. (2001) observaram soroprevalência de $47,63 \%$ em bovinos no Município de Garanhuns.

Altas porcentagens também foram verificadas na região Norte do país por Aguiar et al. (2006), que encontraram soroprevalência de 52,8\% em bovinos no Município de Monte Negro, Rondônia.

A prevalência de 64,81\% (61,10\% - 68,35\%) em rebanhos encontrada no presente estudo revela-se bastante alta e assemelha-se à encontrada por Castro et al. (2008), que observaram taxa de 71,3\% (68,5\% - 74\%) entre as propriedades analisadas no Estado de São Paulo. Maior prevalência em rebanhos foi revelada no estudo de Oliveira et al. (2009) no Estado da Bahia, 77,93\% (75,73\% - 79,99\%). Ainda no Nordeste, Oliveira et al. (2001) encontraram pelo 
menos um animal sororreagente em todas as propriedades analisadas no Município de Garanhuns, Pernambuco.

$\mathrm{Na}$ região Centro-Oeste foi observado prevalência de focos superior à encontrada no presente estudo no Estado do Maranhão, onde Figueiredo et al. (2009) relataram prevalência de 90,4\% (85,8\% - 95,1\%) de sororreação a Leptospira spp. nos rebanhos bovinos analisados no Estado do Mato Grosso do Sul.

Resultados bastante elevados de prevalência de focos também foram encontrados por Homem et al. (2001) no Município de Uruará, Pará, onde 97\% (90,9\% - 99,5\%) dos rebanhos estudados foram considerados positivos. Ainda no Norte do país, no Município de Monte Negro, Rondônia, Aguiar et al. (2006) relataram prevalência de 95,3\% $(88,5 \%-98,7 \%)$ em rebanhos. Ambos os estudos na região Norte do país tiveram resultados de prevalência de focos de leptospirose superiores ao encontrado no presente estudo no Estado do Maranhão.

Ressalta-se a elevada prevalência de focos de leptospirose encontrada no Estado do Maranhão, sendo necessária a imediata elaboração de medidas de prevenção e controle dessa enfermidade, para que o estado consiga atingir melhores indicadores de produção animal, podendo equiparar-se a regiões mais produtivas do país e apresentar bons resultados como estado produtor agropecuário no cenário internacional.

As sorovariedades de Leptospira spp. mais encontradas pela SAM no presente trabalho foram Hardjo $(24,32 \%)$, Wolffi $(22,00 \%)$, Patoc $(12,42 \%)$, Shermani $(8,85 \%)$, Grippotyphosa $(8,21 \%)$ e Hebdomadis $(7,35 \%)$. A prevalência das sorovariedades Wolffi e Hardjo em fêmeas bovinas foi de 20,52\% (17,94\% - 23,37\%), e a prevalência dessas sorovariedades em rebanhos do Estado do Maranhão foi de $38,18 \%(34,66 \%-41,84 \%)$.

Embora com prevalências distintas, há concordância quanto à predominância das sorovariedades Hardjo e Wolffi, com os achados de diversos estudos, o que reforça a importância dessas sorovariedades infectando bovinos e acarretando prejuízos econômicos por ocasionarem problemas reprodutivos nesses animais. Favero et al. (2001), em levantamento realizado em 21 estados brasileiros, encontraram 51,5\% de Hardjo e 24,2\% de Wolffi. Lilenbaum \& Souza (2003), no Rio de Janeiro, obtiveram 43,8\% de reações a Hardjo e 24,7\% a Wolffi. Em Minas Gerais, Araújo et al. (2005) encontraram 19,7\% de Hardjo e 13,2\% de Wolffi. No Município de Pirassununga, SP, Martins (2005) encontrou a sorovariedade Hardjo como a mais frequente em bovinos $(43,4 \%)$, seguida da sorovariedade Wolffi $(11,8 \%)$. No Estado da Paraíba, a sorovariedade Hardjo foi a mais frequente, com 16,05\% das reações positivas (Lage et al. 2007). Em São Paulo, Castro et al. (2008) relataram as sorovariedades Hardjo (46\%) e Wolffi (21\%) como as mais presentes. Figueiredo et al. (2009) encontraram como mais frequentes as sorovariedades Hardjo (65,6\%) e Wolffi (12,3\%). Oliveira et al. (2009) revelaram a sorovariedade Hardjo como a mais frequente, ocorrendo em 14,95\% dos animais analisados.

No presente estudo, embora haja predominância das sorovariedades Hardjo e Wolffi, não deve ser descartada a possibilidade de reação cruzada na soroaglutinação mi- croscópica, pois essas sorovariedades pertencem ao mesmo sorogrupo, sejroe (Costa et al. 1998).

Castro et al. (2008) ressaltaram a importância de possuir ampla composição da bateria antigênica, pois sorovariedades como Shermani, Hebdomadis e Autumnalis, muitas vezes não presentes em baterias de exames de rotina, podem estar presentes infectando rebanhos.

Além da constante presença da sorovariedade Hardjo, cuja transmissão usualmente ocorre entre bovinos, em alguns rebanhos ou regiões podem ocorrer infecções acidentais por outras sorovariedades, cuja transmissão indireta está associada ao contato com o ambiente contaminado por Leptospira spp. oriunda de espécies selvagens ou de outras espécies domésticas. Cervídeos, capivaras e outras espécies selvagens podem atuar como reservatórios de Leptospira spp. para os rebanhos ao encontrar o habitat satisfatório (Castro et al. 2008).

O grande número de sorovariedades de Leptospira spp. encontrado no Estado do Maranhão, Patoc, Shermani, Grippotyphosa e Hebdomadis, Tarassovi, Autumnalis, Pyrogenes, Pomona, Copenhageni, Australis, Canicola, Castellonis, Icterohaemorrhagiae, Panama, Sentot e Andamana, além da Hardjo e da Wolffi, reforça a suspeita da presença frequente e intensa de animais selvagens de vida livre nas propriedades rurais. Existem trabalhos que demonstram o grande número de sorovariedades acometendo animais selvagens de vida livre.

Silva et al. (2010) observaram que gambás (Didelphis albiventris) e cervídeos podem ser reservatórios das sorovariedades Patoc, Autumnalis, Icterohaemorrhagiae, Andamana e Canicola para animais domésticos como bovinos, caprinos, ovinos, suínos, equinos e cães. Sorovariedades acidentais como Patoc, Shermani, Hebdomadis, Autumnalis, Pyrogenes, Australis, Castellonis, Sentot e Andamana, detectadas no presente estudo e cujas descrições são relacionadas com animais silvestres (Santa Rosa et al. 1975, Santa Rosa et al. 1980), levantam a suspeita do envolvimento dessas espécies da fauna selvagem como reservatórios dessas sorovariedades para os bovinos.

Ainda em relação aos marsupiais, os gambás (Didelphis albiventris, Didelphis aurita, Didelphis marsupialis) são reservatórios de várias sorovariedades, tais como Ballum, Bataviae, Icterohaemorrhagiae, Szwajizam e Grippotyphosa (Cubas et al. 2007). Silva et al. (2010) encontraram as sorovariedades Autumnalis, Patoc e Icterohaemorrhagiae em gambás (Didelphis albiventris) e as sorovariedades Autumnalis, Andamana, Icterohaemorrhagiae, Patoc e Canicola em cervídeos.

Souza-Junior et al. (2006), pesquisando anticorpos da classe IgM contra Leptospira interrogans em animais silvestres de vida livre do Estado do Tocantins, observaram que $16,1 \%$ (46 de 286) das amostras de sangue de macacos-prego (Cebus apella) foram positivas às sorovariedades Pomona, Brasiliensis, Mini, Swajizak, Grippothyposa, Sarmin, Fluminense, Autumnalis, Hebdomandis, Guaratuba, Javanica e Icterohaemorrhagiae; entre as 82 amostras de sangue de bugios (Alouatta caraya), duas $(2,4 \%)$ foram positivas às sorovariedades Mangus e Fluminense. Das $31 \mathrm{de}$ quatis (Nasua nasua), quatro (12,9\%) foram positivas às 
sorovariedades Javanica e Fluminense. Duas das 10 (20\%) amostras de cachorros-do-mato (Cerdocyon thous) foram positivas às sorovariedades Brasiliensis e Fluminense.

Em relação à ordem Rodentia, o rato-de-esgoto (Rattus norvegicus) é importante reservatório da sorovariedade Icterohaemorrhagiae, sorovariedade esta que também é frequentemente encontrada no preá (Cavia aperae azarae). 0 rato-d'água (Nectomys squamipes) é o principal reservatório da sorovariedade Australis. Já em capivaras (Hydrochaeris hidrochaeris), as sorovariedades Hardjo, Pomona e Grippotyphosa são as mais vistas. Em cutias (Dasyprocta spp.), observa-se predominantemente a sorovariedade Castellonis (Cubas et al. 2007).

É importante destacar que a sorovariedade Icterohaemorrhagiae, de grande relevância para a saúde pública (Brasil 2005) e frequententemente isolada de roedores (Acha \& Szyfres 2001), teve pouca ocorrência em fêmeas bovinas no Estado do Maranhão (0,48\%). Resultados diferentes foram obtidos por Juliano et al. (2000) em Goiânia e por Figueiredo et al. (2009) no Mato Grosso do Sul, os quais relataram ocorrência de $20,6 \%$ e $5,8 \%$ dessa sorovariedade entre os animais reagentes, respectivamente.

Outras sorovariedades encontradas no presente estudo, além da Icterohaemorrhagiae, como Patoc, Shermani, Grippotyphosa e Hebdomadis, podem representar importância para a saúde pública caso o ambiente esteja bastante contaminado com urina de animais infectados e a dose infectante em uma população exposta seja alta. Além disso, uma condição pré-existente de imunodeficiência em um indivíduo ou em um grupo de indivíduos expostos a ambientes contaminados por essas demais sorovariedades pode gerar, nesses indivíduos, um quadro clínico grave, mesmo sendo baixa a dose infectante.

0 circuito III foi o que apresentou menor prevalência de leptospirose em fêmeas bovinas $(15,53 \%$; IC $95 \%=$ $11,05-21,39)$ e em rebanhos $(30,33 \%$; IC $95 \%=22,79$ - 39,10). Também apresentou menor prevalência das sorovariedades de Leptospira spp. Hardjo e/ou Wolffi em fêmeas $(2,63 \%$; IC $95 \%=0,96-6,99)$ e em rebanhos $(3,28 \%$; IC $95 \%=1,23-8,46)$. Esse achado se deve, possivelmente, às características climáticas desse circuito, pois é uma região onde ocorrem longas estiagens. O circuito III corresponde a uma região do Estado do Maranhão na qual se observam características marcantes do bioma Caatinga, bioma este que, pelos baixos índices pluviométricos e pela elevada temperatura média anual, não fornece boas condições à sobrevivência de Leptospira spp. Já nos circuitos I, II e IV observam-se características dos biomas Zona Costeira, Amazônia e Cerrado, respectivamente, regiões com índices pluviométricos mais altos. Por isso, são necessárias estratégias de controle mais eficientes para a leptospirose nesses três circuitos, principalmente em locais com saneamento precário e presença constante de animais selvagens reservatórios, especialmente roedores, espécies caracterizadas como fatores de risco da enfermidade no presente estudo.

Há estudos relacionando a ocorrência de casos de leptospirose com as condições do ambiente. Num estudo da distribuição espacial da leptospirose no Rio Grande do Sul,
Barcellos et al. (2003) identificaram áreas de maior risco e possíveis componentes ecológicos da transmissão da leptospirose. As maiores taxas de ocorrência da doença foram verificadas em áreas litorâneas, de baixa altitude e uso do solo predominantemente agrícola, de lavoura irrigada. Esses locais, segundo o estudo, possuem características ecológicas favoráveis à proliferação de roedores, favorecendo a transmissão de Leptospira spp.

Com o objetivo de analisar os fatores de risco associados à soropositividade para leptospirose em matrizes suínas, Delbem et al. (2004) apontaram como fatores de risco a existência de áreas alagadiças próximas às instalações, o uso de bebedouro tipo canaleta e a inexistência de higienização do reservatório de água.

No presente estudo, a variável presença de equinos foi caracterizada como fator de risco de leptospirose. Esse resultado reforça a importância epidemiológica dos herbívoros como fonte de infecção de Leptospira spp., devido a composição levemente alcalina da urina desses animais, característica que contribui para maior sobrevivência dessa bactéria no ambiente, aumentando suas chances de infectar um hospedeiro suscetível (Faine 1999).

A presença de mais de 32 animais em rebanhos bovinos $(\mathrm{OR}=2,069$; IC 95\% = 1,312 - 3,262; $\mathrm{p}<0,002)$ foi identifica$\mathrm{da}$, no presente estudo, como fator de risco de leptospirose por qualquer sorovariedade. Quanto maior é um rebanho, maiores são as chances de um agente etiológico alcançar um hospedeiro suscetível, ou seja, maior é a transmissão do agente. Resultados semelhantes foram encontrados por Oliveira et al. (2010), que, ao analisarem possíveis fatores de risco de leptospirose em fêmeas bovinas em idade reprodutiva no Estado da Bahia, concluíram que a presença de mais de 28 fêmeas bovinas com idade igual ou superior a 24 meses (OR $=2,11$; IC $95 \%=1,27-3,52$; $p<0,001)$ é fator de risco de leptospirose por qualquer sorovariedade. Da mesma maneira, Martins (2005) concluiu que ter mais de 21 cabeças no rebanho $(O R=14,354$; IC $95 \%=1,535-134,215)$ é fator de risco para leptospirose por qualquer sorovariedade.

A presença de capivaras (Hydrochaeris hydrochaeris) também foi identificada como fator de risco de leptospirose $(\mathrm{OR}=3,817$; IC 95\% $=1,110-13,132 ; \mathrm{p}=0,034)$ no presente estudo. No Brasil, investigações sorológicas para detecção de infecção por leptospiras (Nogueira et al. 1997, Ito et al. 1998, Marvulo et al. 2002, Shimabukuro 2006) e isolamento de leptospiras (De Paula et al. 2001) têm sido realizados em capivaras de vida livre a fim de demonstrar seu papel como reservatório dessas bactérias. 0 desequilíbrio ecológico aumenta a importância das capivaras como reservatório de Leptospira spp., pois mudanças no seu habitat natural ocasionam a invasão dessa espécie a lavouras e à periferia de áreas urbanas (Cueto et al. 2000).

Oliveira et al. (2010) também identificaram a participação de animais selvagens como fator de risco para a enfermidade. Nesse trabalho, constataram que a presença de cervídeos foi fator de risco de leptospirose $(\mathrm{OR}=2,02$; IC $95 \%=1,16-3,50 ; \mathrm{p}=0,01$ ).

0 questionário elaborado por técnicos da AGED-MA e do MAPA para brucelose foi utilizado no presente estudo para a análise de possíveis fatores de risco de leptospirose 
bovina no Estado do Maranhão. Entre as variáveis, é importante destacar presença de animais silvestres, presença de cervídeos, presença de capivaras e presença de outros silvestres. É sabida a importância de espécies selvagens de vida livre como reservatórios de um grande número de sorovariedades de Leptospira spp. Além disso, é importante destacar que presença de capivaras foi fator de risco neste estudo, porém presença de animais silvestres e presença de outros silvestres não foram consideradas fatores de risco. Isso se deve, muito possivelmente, ao fato de essas duas variáveis serem relacionadas a aves silvestres e não a mamíferos, visto que as aves não são importantes na cadeia epidemiológica da leptospirose e, portanto, o valor de "p" para essas variáveis foi alto, não significativo. Quando se isola uma variável só para roedores, o valor de " $p$ " fica possivelmente significativo devido à grande importância dessas espécies na cadeia epidemiológica da leptospirose.

\section{CONCLUSÕES}

O Estado do Maranhão possui alta prevalência de leptospirose tanto em fêmeas bovinas quanto de focos, e essa prevalência está diretamente relacionada às características climáticas.

Elevadas densidades de animais em um rebanho aumentam as chances de transmissão da leptospirose de uma fonte de infecção a hospedeiros suscetíveis.

Herbívoros têm grande papel como fonte de infecção de Leptospira spp. e animais selvagens de vida livre são reservatórios de leptospirose para animais domésticos.

Há necessidade de mais estudos sobre os prejuízos das diversas sorovariedades de Leptospira spp. para a saúde pública e sobre o papel de animais selvagens de vida livre na cadeia epidemiológica da leptospirose, para que medidas higiênico-sanitárias e de manejo sejam postas em prática e, com isso, prevenir e controlar essa enfermidade de maneira mais eficiente.

\section{REFERÊNCIAS}

Acha P.N. \& Szyfres B. 2001. Zoonosis y enfermedades transmisibles comunes al hombre y a los animales. 3aㅡ ed. Publicación Científica 398, OPS, Washington. 503p.

Aguiar D.M., Gennari S.M., Cavalcante G.T., Labruna M.B., Vasconcellos S.A., Rodrigues A.A.R., Moraes Z.M. \& Camargo L.M.A. 2006. Seroprevalence of Leptospira spp. in cattle from Monte Negro municipality, western Amazon. Pesq. Vet. Bras. 26(2):102-104.

Araújo V.E.M., Moreira E.C., Navega L.A.B., Silva J.A. \& Contreras R.L. 2005. Freqüência de aglutininas anti-Leptospira interrogans em soros sangüíneos de bovinos, em Minas Gerais, de 1980 a 2002. Arq. Bras. Med. Vet. Zootec. 57(4):430-435.

Barcellos C., Lammerhirt C.B., Almeida M.A.B. \& Santos E. 2003. Distribuição espacial da leptospirose no Rio Grande do Sul, Brasil: recuperando a ecologia dos estudos ecológicos. Cad. Saúde Públ. 19(5):12831292.

Blaha T. 1989. Applied Veterinary Epidemiology. Elsevier, Amsterdam, p.95-103.

Brasil 1995. Manual de Leptospirose. $2^{\text {a }}$ ed. Fundação Nacional de Saúde, Ministério da Saúde, Brasília. 98p.

Brasil 2005. Guia de Vigilância Epidemiológica. 6ae ed. Secretaria de Vigilância em Saúde, Ministério da Saúde, Brasília, p.502-520.

Castro V., Azevedo S.S., Gotti T.B., Batista C.S.A., Gentili J., Morais Z.M., Souza G.O., Vasconcellos S.A. \& Genovez M.E. 2008. Soroprevalência da lep- tospirose em fêmeas bovinas em idade reprodutiva no Estado de São Paulo, Brasil. Arqs Inst. Biológico, São Paulo, 75(1):3-11.

Cervantes L.P.M., Puebla M.A.C., Rosas D.G., Serrania N.R. \& Barranca J.I.T. 2002. Estudio serológico de leptospirosis bovina en México. Revta Cub. Med. Trop. 54(1):24-27

Costa M.C.R., Moreira E.C., Leite R.C. \& Martins N.R.S. 1998. Avaliação da imunidade cruzada entre Leptospira hardjo e L. wolffi. Arq. Bras. Med. Vet. Zootec. 50(1):11-17.

Cubas Z.S., Silva J.C.R. \& Catão-Dias J.L. 2007. Tratado de Animais Selvagens: medicina veterinária. Roca, São Paulo. 1376p.

Cueto G.R., Allekotte R. \& Kravetz F.O. 2000. Scurvy in capybaras bred in captivity in Argentine. J. Wildl. Dis. 36:97-101.

De Paula C.D., Marvulo M.F.V. \& Miraglia F. 2001. Isolamento de leptospira em capivaras (Hydrochaeris hydrochaeris) de vida livre. Anais V Congresso e X Encontro da Associação Brasileira de Veterinários de Animais Selvagens, São Paulo, SP, s/p. (Resumo)

Delbem A.C.B., Freire R.L., Silva C.A., Müller E.E., Dias R.A., Neto J.S.F. \& Freitas J.C. 2004. Fatores de risco associados a soropositividade para leptospirose em matrizes suínas. Ciência Rural 34(3):847-852.

Faine S. 1999. Leptospira and Leptospirosis. $2^{\text {nd }}$ ed. MedSci, Melbourne. 272p.

Favero M., Pinheiro S.R., Vasconcellos S.A., Morais Z.M., Ferreira F. \& Ferreira-Neto J.S. 2001. Leptospirose bovina: variantes sorológicas predominantes em colheitas efetuadas no período de 1984 a 1997 em rebanhos de 21 estados do Brasil. Arqs Inst. Biológico, São Paulo, 68(2):29-35.

Figueiredo A.O., Pellegrin A.O., Gonçalves V.S.P., Freitas E.B., Monteiro L.A.R.C., Oliveira J.M. \& Osório A.L.A.R. 2009. Prevalência e fatores de risco para a leptospirose em bovinos de Mato Grosso do Sul. Pesq. Vet. Bras. 29(5):375-381.

Guimarães M.C., Côrtes J.A. \& Vasconcellos S.A. 1982/1983. Epidemiologia e controle de leptospirose em bovinos. Papel do portador e seu controle terapêutico. Comun. Cient., Fac. Med. Vet. Zootec., São Paulo, 6/7:21-34.

Homem V.S.F., Heinemann M.B., Moraes Z.M., Vasconcellos S.A., Ferreira F. \& Ferreira-Neto J.S. 2001. Estudo epidemiológico da leptospirose bovina e humana na Amazônia oriental brasileira. Revta Soc. Bras. Med. Trop. 34(2):173-180.

Ito F.H., Vasconcelos S.A., Bernardi F., Nascimento A.A., Labruna M.B. \& Arantes I.G. 1998. Evidencia sorológica de brucelose e leptospirose e parasitismo por ixodídeos em animais silvestres do Pantanal Sul-Matogrossense. Ars Vet. 13:302-310.

Juliano R.S., Chaves N.S.T., Santos C.A., Ramos L.S., Santos H.Q., Meireles L.R., Gottschalk S. \& Corrêa-Filho R.A.C. 2000. Prevalência e aspectos epidemiológicos da leptospirose bovina em rebanho leiteiro da microrregião de Goiânia/GO. Ciência Rural 30(5):857-862.

Lage A.P., Leite R.M.H., Thompson J.A., Bandeira D.A., Herrman G.P., Moreira E.C. \& Gonçalves V.S.P. 2007. Serology for Leptospira sp. in cattle of the State of Paraíba, Brazil. Arqs Inst. Biológico, São Paulo, 74(3):185-190.

Langoni H., Meireles L.R., Gottschalk S., Cabral K.G. \& Silva A.V. 2000. Perfil sorológico da leptospirose bovina em regiões do Estado de São Paulo. Arqs Inst. Biológico, São Paulo, 67(1):37-41.

Lilenbaum W. \& Souza G.N. 2003. Factors associated with bovine leptospirosis in Rio de Janeiro, Brazil. Res. Vet. Sci. 75:249-251.

Madruga C.R., Aycardi E. \& Putt N. 1980. Freqüência de aglutininas anti-leptospira em bovinos de corte na região Sul de Cerrado do Estado de Mato Grosso. Arq. Esc. Vet., Belo Horizonte, 32(2):245-249.

Martins L.S. 2005. Situação epidemiológica da leptospirose bovina, canina e humana na área rural do município de Pirassununga, SP. Tese de Doutorado em Medicina Veterinária, Faculdade de Medicina Veterinária e Zootecnia, Universidade de São Paulo, SP. 79p.

Marvulo M.F.V., Paula C.D., Ferreira P.M., Morais Z.M., Delbem A.C.B., Fávero A.C.M., Miraglia F., Castro V., Genovez M.E., Ferraz K., Verdade L.M., Fellipe P.A.N., Ferraz E., Penteado M., Ferreira Neto J.S., Ferreira F. \& Vasconcellos S.A. 2002. Detection of Leptospira in two free living populations of capybaras (Hydrochaeris hydrochaeris) from São Paulo state, Brazil. Proc. $3^{\text {rd }}$ Scient. Meet. Int. Lepospirosis Society, Barbados, s/p. (Abstract) 
Nogueira M.F., Langoni H., Lavorenti A., Gimenes S.M. \& Nogueira Filho S.L.G. 1997. Detecção de anticorpos anti-Leptospira spp. e anti-Brucella abortus em capivaras (Hydrochaeris hydrochaeris). Anais XIX Congresso Brasileiro de Microbiologia, Rio de Janeiro, RJ, s/p.

Noordhuizen J.P.T.M., Frankena K., Van Der Hoofd C.M. \& Graat E.A.M. 1997. Application of quantitative methods in veterinary epidemiology. Wageningen Press, Wageningen. 445p.

Oliveira A.A.F., Mota R.A., Pereira G.C., Langoni H., Souza M.I., Navegantes W.A. \& Sa, M.E.R. 2001. Seroprevalence of bovine leptospirosis in Garanhuns municipal district, Pernambuco State, Brazil. Onderstepoort J. Vet. Res. 68:275-279.

Oliveira F.C.S., Azevedo S.S., Pinheiro S.R., Viegas S.A.R.A., Batista C.S.A., Coelho C.P., Moraes Z.M., Souza G.O., Gonçalves A.P., Almeida C.A.S. \& Vasconcellos S.A. 2009. Soroprevalência de leptospirose em fêmeas bovinas em idade reprodutiva no Estado da Bahia. Arqs Inst. Biológico, São Paulo, 76(4):539-546.

Oliveira F.C.S., Azevedo S.S., Pinheiro S.R., Batista C.S.A., Moraes Z.M., Souza G.O., Gonçalves A.P. \& Vasconcellos S.A. 2010. Fatores de risco para a leptospirose em fêmeas bovinas em idade reprodutiva no Estado da Bahia, Nordeste do Brasil. Pesq. Vet. Bras. 30(5):398-402.

Pellegrin A.O. \& Sereno J.R.B. 1994. Leptospirose e sua relação com fertilidade em um grupo de matrizes neloradas no Pantanal, sub-região da Nhecolândia. Anais 23ํㅡㄹ Congresso Brasileiro de Medicina Veterinária, Olinda, p.189. (Resumo)

Pellegrin A.O., Sereno J.R.B. \& Figueiredo, J.O. 1992. Levantamento sorológico de aglutininas anti-leptospira em bovinos da sub-região da Nhecolândia, Pantanal Sul-Matogrossense. Anais 22ํㅡㄹ Congresso Brasileiro de Medicina Veterinária, Curitiba p.145.

Pellegrin A.O., Guimarães P.H.S., Sereno J.R.B., Figueiredo J.P., Lage A.P., Moreira E.C. \& Leite R.C. 1999. Prevalência da leptospirose em bovinos do Pantanal Mato-Grossense. Comum. Téc. 22, Embrapa Pantanal, Corumbá, p.1-9.
Sampaio I.B.M. 2007. Estatística Aplicada à Experimentação Animal. $3^{a}$ ed., FEP-MVZ, Belo Horizonte. 264p.

Santa Rosa C.A., Sulzer C.R., Giorgi W., Silva A.S., Yanaguita R.M. \& Lobão A.0. 1975. Leptospirosis in wildlife in Brazil: Isolation of a new serotype in Pyrogenes group. Am. J. Vet. Res. 36:1363-1365.

Santa Rosa C.A., Sulzer C.R., Yanaguita R.M. \& Silva A.S. 1980. Leptospirosis in wildlife in Brazil: Isolation of serovars Canicola, Pyrogenes and Grippotyphosa. Intern. J. Zoonoses 7:40-43.

Shimabukuro J.S. 2006. Estudo da soroprevalência de Leptospira spp. em capivaras (Hydrochaeris hydrochaeris) na bacia hidrográfica do Alto Tietê, SP. Dissertação de Mestrado em Epidemiologia Experimental Aplicada às Zoonoses, Universidade de São Paulo, São Paulo, SP. 50p.

Silva F.J., Mathias L.A., Magajevski F.S., Werther K., Assis N.A. \& Girio R. J.S. 2010. Anticorpos contra Leptospira spp. em animais domésticos e silvestres presentes no campus universitário da FCAV, Unesp, Jaboticabal/ SP. Ars Vet. 26(1):17-25.

Souza-Junior M.F.S., Lobato Z.I.P., Lobato F.C.F., Moreira E.C., Oliveira R.R., Leite G.G., Freitas T.D. \& Assis R.A. 2006. Presença de anticorpos da classe IgM de Leptospira interrogans em animais silvestres do Estado do Tocantins. Revta Soc. Bras. Med. Trop. 39(3):292-294.

Sulzer C.R. \& Jones W.L. 1980. Leptospirosis: method in laboratory diagnosis. Center for Diseases Control, Atlanta.

Thompson J.A., Leite R.M.H., Gonçalves V.S.P., Leite R.C., Bandeira D.A., Herrmann G.P., Moreira, E.C., Prado P.E.F., Lobato Z.I.P., Brito C.P.T. \& Lage A.P. 2006. Spatial hierarchical variances and age covariances for seroprevalence to Leptospira interrogans serovar hardjo, BoHV-1 and BVDV for cattle in the State of Paraíba, Brazil. Prev. Vet. Med. 76:290301.

Thrusfield M. 1995. Veterinary Epidemiology. $2^{\text {nd }}$ ed. Blackwell Science, Cambridge. $479 \mathrm{p}$. 